American J. of Engineering and Applied Sciences 3 (1): 193-200, 2010

ISSN 1941-7020

(C) 2010 Science Publications

\title{
Identification of Instabilities of the Chip Formation and It's Prediction Model During End Milling of Medium Carbon Steel (S45C)
}

\author{
${ }^{1}$ Md. Anayet U Patwari, ${ }^{1}$ A.K.M. Nurul Amin and ${ }^{2}$ Waleed Faris \\ ${ }^{1}$ Department of Manufacturing and Materials Engineering, \\ ${ }^{2}$ Department of Mechanical Engineering, Faculty of Engineering, \\ International Islamic University Malaysia, Kuala Lumpur, P.O. Box 10, 50728, Malaysia
}

\begin{abstract}
Problem statement: Chip shape and size varied widely in machining operations. Undesirable chip formation had a detrimental effect on surface finish, work-piece accuracy, chatter and tool life. Approach: This study included the findings of an experimental study on the instabilities of the chip formation and development of a mathematical model based on statistical approach for the prediction of the instability of chip formation during the machining of medium carbon steel (S45C). Results: It has been identified that the chip formation process has a discrete nature, associated with the periodic shearing process of the chip. Typical instabilities of periodic nature, in the form of primary and secondary saw/serrated teeth, which appear at the main body and free edge of the chip respectively, have been identified. Mechanisms of formation of these teeth have been studied and the frequencies of their formation have been determined under various machining conditions. Small Central composite design was employed in developing the chip serration frequency model in relation to primary cutting parameters by Response Surface Methodology (RSM). Conclusion/Recommendations: The mathematical model for the chip serration frequency has been developed, in terms input cutting parameters (cutting speed, feed and depth of cut) in end milling of S45C steel using TiN inserts under full immersion. The adequacy of the predictive model was verified using ANOVA at 95\% confidence level.
\end{abstract}

Key words: Chip serration frequency, end milling, RSM model

\section{INTRODUCTION}

High metal removal rate with a desired quality of surface finish, which is the ultimate objective in machining, depends on a large number of factors, that include chip-tool interaction, cutting temperature, wear mechanism and wear rate, cutting force, chatter and dynamics behavior of the machine tool system. Problems with surface finish, work-piece accuracy, chatter and tool life can be caused even by minor changes in the chip formation process. At lower cutting speeds the chip is often discontinuous, while it becomes serrated as the cutting speeds are increased. In metal cutting, the present tendency is towards achieving increased material removal rates with very reliable machining processes, where the predictability of surface finish, work-piece accuracy, chatter and tool life are of prime importance. But to maintain stable machining, much attention must also be paid to the formation of the desired type of chip and chip controls to facilitate its easy removal. This is because the chip formation and breaking aspect is very significant in machining.

One of the restrictions limiting large material removal rates is the tendency of the machine tool to chatter. Trent (2002); Talantov et al. (1980) and Amin (1983) considered the formation of chips with serrated teeth to be the primary cause of chatter. Talantov et al. (1980) and Amin (1983) have observed that chatter arising during turning is a result of resonance, caused by mutual interaction of the vibrations due to serrated elements of the chip and the natural vibrations of the system components, e.g., the spindle and the tool holder (Talantov et al., 1980; Amin, 1983). Much research study has been done on the chip formation in turning, drilling and face milling. Komanduri (1981) and Komanduri and Schroeder (1982) has made some remarkable progress in the research of chip segmentation and instability in chip formation. Nevertheless it appears that very few works have been done to investigate the nature of chip formation in end

Corresponding Author: Anayet U. Patwari, Department of Manufacturing and Materials Engineering, International Islamic University Malaysia, Kuala Lumpur, P.O. Box 10, 50728, Malaysia 
milling because of its complexity and geometrical difficulty. Toenshoff (1969) proposed the basic chip formation mechanism as "adiabatic shear" at high cutting speed. Changing speed during the machining process or finding an optimum speed are the commonest tactics to avoid chatter in milling. Various methods have been proposed by many researchers (Tlusty and Ismail, 1983; Smith and Tlusty, 1987; Elbestawi, 1997; Altintas, 1991; Minis, 1993) in this regard. It is found in some instances that by changing the spindle speed as well as the feed rate it is possible to increase the maximum cutting depth. At a certain spindle speed, it is usually found that a larger maximum stable cutting depth is obtained by applying a higher feed rate. As a result, the chip formation is improved and the desired stable chip is obtained. Amin and Talantov (1986) earlier established that the instability of chip formation could be lowered by preheating the work material during turning. Ning et al. (2001) indicates that chatter could be reliably recognized by analysis of the chips. Ekinovic et al. (2002) mentioned in their study that cutting speed has significant effect on chip formation models. Similar influence of the cutting speed on the chip structure and chip compression ratio was revealed in the experiments conducted by Tonshoff et al. (1999). As the chip formation process appears to be cyclic, its frequency is of interest. The frequency of chip formation can be measured by calculating the number of teeth produced in unit time as proposed by Talantov (1984). Lindberg and Lindstrom (1983) proposed direct measurement of this frequency using a digital spectrum analyzer. Astakhov and Shvets (2001) studied the chip formation frequency of different work materials at the same cutting regimes and dimensions of the work-pieces. The results of this study revealed that the frequency of the chip formation process primarily depends on the cutting speed and on the work material. The cutting feed and the depth of cut have very small influence on this frequency. The results obtained also show that when the dynamic experiments are conducted properly, i.e., when the noise (for example, due to the misalignment of the work-piece and other machining system inaccuracies) is eliminated from the system response, the amplitudes of peak at the frequency of chip formation are the largest. Unfortunately, this fact is not considered in the dynamic analysis of the machining systems where the cutting process is practically the main source of vibration. Amin (1982) concluded based his investigations on mainly three work materials-AISI 1045, a titanium alloy and heat resistant alloy steel, that with the increase in cutting speed during turning, there is an inherent instability in metal cutting process which lead to the formation of secondary serrated teeth with a certain frequency at the free edge of the chip (away from the tool nose). The side teeth were termed as 'secondary serrated teeth' to distinguish them from the primary serrated teeth that appear in the entire cross section of the chip during machining of titanium and its alloys. It was also observed that as the cutting speed was increased, the secondary serrated teeth extended to the whole cross-section of the chip to form the primary serrated teeth in the chip. It was observed that the frequency of chip serration increased with the cutting speed and was higher for harder materials tike, titanium alloys and hardened steel, compared to softer and more ductile materials, like, plain carbon steels. The phenomenon of chip serration during the machining of Ti-6Al-4V was also observed by Komenduri and Von Turkovich (1981) who proposed the well-known 'catastrophic shear band' theory. Amin (1982) found that the root cause of chatter lies in the coincidence of the frequency of instability of chip formation and one of the natural frequencies of the machine-spindle-tool system. The instability of chip was found to be due to the formation of cyclic chip, widely known as the serrated chips. In this study the instability of the chip formation processes has been identified and calculated in terms of serration frequency and a mathematical model has been developed which will be helpful to predict the chatter formation during machining of medium carbon steel within this specified ranges.

\section{MATERIALS AND METHODS}

Equipment: Cutting tests was conducted mainly on Vertical Machining Center (VMC ZPS, Model: 1060) powered by a $30 \mathrm{KW}$ motor with a maximum spindle speed of $8000 \mathrm{rpm}$. Figure 1 shows the experimental set up cutting test conditions on end milling for machining of medium carbon steel with TiN inserts.

Tool (inserts)-SANDVIK grade PM1030 Insert code: R390-11 T3 08E-PL, Insert coating material: Carbide, Working condition: light to medium milling. For insert geometry refer to Fig. 2 and Table 1.

Analysis of chips produced in end milling: The chips formed during end milling were mainly investigated and it has been found that at some specific cutting conditions chip formation presents extreme cases of secondary and primary chip serration.

Table 1: Insert geometry values

\begin{tabular}{lllllll}
\hline $\mathrm{L}$ & $\mathrm{iW}$ & $\mathrm{d}_{1}$ & $\mathrm{~s}$ & $\mathrm{~b}_{\mathrm{s}}$ & $\mathrm{r}_{\varepsilon}$ & $\alpha_{\mathrm{n}}{ }^{\circ}$ \\
\hline 11 & 6.8 & 2.8 & 3.59 & 1.2 & 0.8 & 21 \\
\hline
\end{tabular}




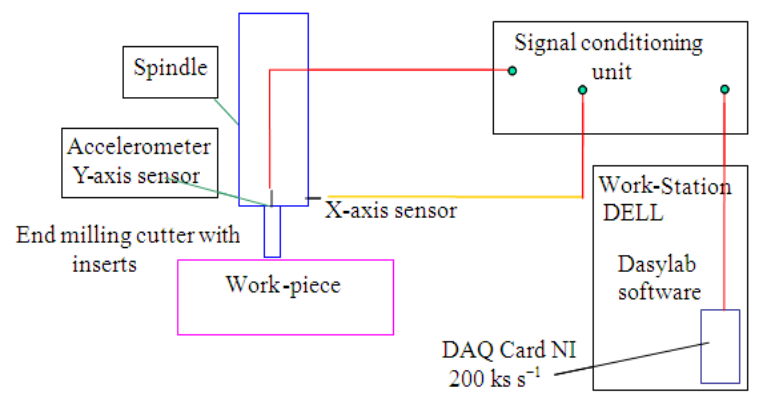

Fig. 1: Experimental set up for end milling
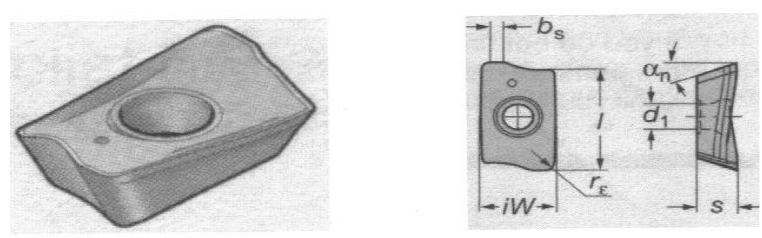

Fig. 2: Insert shape and geometry

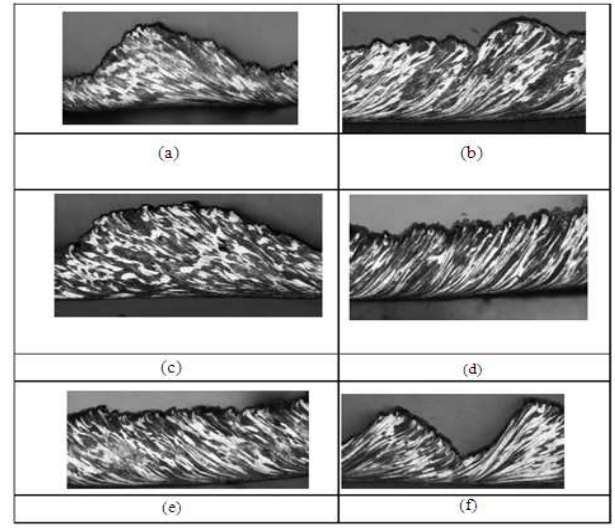

Fig. 3: Chip morphology length-wise sections of the chips produced under different cutting conditions, (a): CS: $218.5 \mathrm{~m} \mathrm{~min}^{-1}$ DOC: 2.2 mm, Feed: $0.05 \mathrm{~mm} /$ tooth, (b): CS: $218.5 \mathrm{~m}$ $\mathrm{min}^{-1}$ DOC: $1.15 \mathrm{~mm}$, Feed: $0.16 \mathrm{~mm} / \mathrm{tooth}$, (c): CS: $114.25 \mathrm{~m} \mathrm{~min}^{-1}$ DOC: $2.2 \mathrm{~mm}$, Feed: $0.16 \mathrm{~mm} /$ tooth (d): CS: $114.25 \mathrm{~m} \mathrm{~min}^{-1}$ DOC: $1.15 \mathrm{~mm}$, feed: $0.05 \mathrm{~mm} /$ tooth, (e): CS: $100 \mathrm{~m} \mathrm{~min}^{-1}$ DOC: $1.59 \mathrm{~mm}$, Feed: 0.089 $\mathrm{mm} /$ tooth, (f): CS: $158 \mathrm{~m} \mathrm{~min}^{-1}$ DOC: $2.51 \mathrm{~mm}$, Feed: $0.089 \mathrm{~mm} /$ tooth

Typical SEM pictures and micro-sections of chips formed under various conditions were studied. Firstly, the chip at different cutting conditions were collected and chip specimens were prepared, polished and etched for viewing under the microscope to capture the structure of the chip. General observation shows that chip has the primary saw teeth.

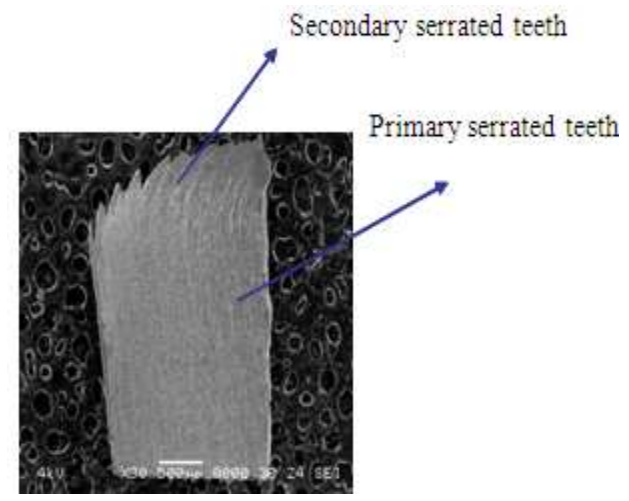

Fig. 4: SEM view of the chip with serrated elements

The primary saw teeth are produced due to the alternative phase compression and adiabatic shear process resulting in fluctuation of the force exerted on the tool. Some sample picture length-wise sections of the chips produced under different cutting conditions are shown in Fig. 3 at different cutting conditions. The chips formed in end milling are serrated in nature.

The primary serrated teeth are formed at main body of the chip at almost equal pacing. The sectional views of serrated elements show the directional flow of deformed grains. Secondary serrated elements are also found to form comprising several primary serrated teeth at the free edge of the chip (Fig. 3). Primary serrated teeth formation is the outcome of the inherent discrete behavior of the chip formation process. Cross sectional view of the chip reveal that the chip element undergoes alternate phases of compression and shear for the formation of saw teeth.

Formulation of chip analysis-chip serration frequency: In order to have a close look at the chip to identify its morphology and inspect the presence of the primary and/or the secondary serrated teeth and any other type of instability that might be present in the outer view of the chip, the latter was viewed under a Scanning Electron Microscope (SEM) as shown in Fig. 4. The frequency of the secondary serrated teeth formation, $F_{c}$, in the cases of milling operation was calculated knowing the length of the portion of the chip in the SEM pictures, L, the coefficient of chip shrinkage, $\mathrm{K}$ (determined by dividing the uncut chip length by the actual chip length), cutting speed, $\mathrm{V}$ $\mathrm{m} \mathrm{min}{ }^{-1}$ and the number of secondary serrated teeth, $\mathrm{n}$, observed on the SEM picture; using the following formula (Amin, 1982):

$$
\mathrm{F}_{\mathrm{c}}=1000 \frac{\mathrm{nV}}{60(\mathrm{LK})}[\mathrm{Hz}]
$$


Response surface methodology: RSM is a collection of mathematical and statistical techniques that are useful for the modeling and analysis of problems in which a response of interest is influenced by several variables and the objective is to optimize this response (Montgomery, 1997). RSM also quantifies relationships among one or more measured responses and the vital input factors (Design-Expert Software, 2000). The version 6.0.8 of the Design Expert software was used to develop the experimental plan for RSM. The same software was also used to analyze the data collected using the steps as follows:

- Choose a transformation if desired. Otherwise, leave the option at "None"

- Select the appropriate model to be used. The Fit Summary button displays the sequential F-tests, lack-of-fit tests and other adequacy measures that could be used to assist in selecting the appropriate model

- Perform the Analysis Of Variance (ANOVA), postANOVA analysis of individual model coefficients and case statistics for analysis of residuals and outlier detection

- Inspect various diagnostic plots to statistically validate the model

- If the model looks good, generate model graphs, i.e., the contour and 3D graphs, for interpretation. The analysis and inspection performed in steps stated above will show whether the model is good or otherwise. Very briefly, a good model must be significant and the lack-of-fit must be insignificant. The various coefficient of determination, $\mathrm{R}^{2}$ values should be close to 1 . The diagnostic plots should also exhibit trends associated with a good model and these will be elaborated subsequently

After analyzing each response, multiple response optimization technique was performed, either by inspection of the interpretation plots, or with the graphical and numerical tools provided for this purpose. It was mentioned previously that RSM designs also help in quantifying the relationships between one or more measured responses and the vital input factors. In order to determine if there exist a relationship between the factors and the response variables investigated, the data collected must be analyzed in a statistically sound manner using regression. A regression is performed in order to describe the data collected whereby an observed, empirical variable (response) is approximated based on a functional relationship between the estimated variable, $y_{\text {est }}$ and one or more regressor or input variable $\mathrm{x}_{1}, \mathrm{x}_{2}, \ldots, \mathrm{x}_{\mathrm{i}}$. The least square technique is being used to fit a model equation containing the said regressors or input variables by minimizing the residual error measured by the sum of square deviations between the actual and the estimated responses. This involves the calculation of estimates for the regression coefficients, i.e., the coefficients of the model variables including the intercept or constant term. The calculated coefficients or the model equation need to however be tested for statistical significance. In this respect, the following tests are performed:

- Test for significance of the regression model

- Test for significance on individual model coefficients

- Test for lack-of-fit

Mathematical model on chip serration: Chip serration frequency model for end milling in terms of the parameters can be expressed in general terms as:

$\mathrm{f}_{\mathrm{c}}=\mathrm{D} * \mathrm{~V} * \mathrm{a}^{\mathrm{y}} \mathrm{f}_{\mathrm{z}}^{\mathrm{z}}$

Where:

$\mathrm{f}_{\mathrm{c}}=$ The predicted chip serration frequency $(\mathrm{Hz})$

$\mathrm{V}=$ The cutting speed $\left(\mathrm{m} \mathrm{min}^{-1}\right)$

$\mathrm{f}_{\mathrm{z}}=$ The feed per tooth $(\mathrm{mm} /$ tooth $)$

$\mathrm{a}=$ The axial depth of cut $(\mathrm{mm})$

$\mathrm{D}, \mathrm{x}, \mathrm{y}$ and $\mathrm{z}$ are model parameters to be estimated using the experimental results. To determine the constants and exponents, this mathematical model can be linearized by employing a logarithmic transformation and Eq. 1 can be re-expressed as:

$\ln \mathrm{f}_{\mathrm{c}}=\ln \mathrm{D}+\mathrm{x} \ln \mathrm{V}+\mathrm{y} \ln \mathrm{a}+\mathrm{z} \ln \mathrm{f}_{\mathrm{z}}$

The linear model of Eq. 3 is:

$y=\beta_{0} x_{0}+\beta_{1} x_{1}+\beta_{2} x_{2}+\beta_{3} x_{3}$

Where:

$\mathrm{y}=$ The true response of Chip Serration Frequency on a logarithmic scale $\mathrm{x}_{0}$ $=1$ (dummy variable)

$\mathrm{x}_{1}, \mathrm{x}_{2}$ and $\mathrm{x}_{3}=$ Logarithmic transformations of speed, depth of cut and feed, respectively

$\beta_{0}, \beta_{1}, \beta_{2}$ and $\beta_{3}=$ The parameters to be estimated

Equation 4 can be expressed as:

$\hat{y}_{1}=y-\varepsilon=b_{0} x_{0}+b_{1} x_{1}+b_{2} x_{2}+b_{3} x_{3}$ 
Am. J. Engg. \& Applied Sci., 3 (1): 193-200, 2010

Where:

$\hat{y}=$ The estimated response

$\mathrm{y}=$ The measured Chip Serration Frequency on a logarithmic scale

$\varepsilon=$ Experimental error

$\mathrm{b}=$ Values are estimates of the $\beta$ parameters.

The second-order model can be extended from the first-order model equation as:

$$
\begin{aligned}
\hat{y}_{2}= & y-\varepsilon=b_{0} x_{0}+b_{1} x_{1}+b_{2} x_{2}+b_{3} x_{3}+b_{11} x_{1}^{2} \\
& +b_{22} x_{2}^{2}+b_{33} x_{3}^{2}+b_{12} x_{1} x_{2}+b_{13} x_{1} x_{3}+b_{23} x_{2} x_{3} \ldots
\end{aligned}
$$

where, $\hat{y}_{2}$ is the estimated response based on the second order model. Analysis of variance is used to verify and validate the model.

Experimental details: In this study, cutting tests were carried out for end milling in dry conditions on vertical machining centre with $20 \mathrm{~mm}$ diameter tool holder fitted with a single coated TiN insert with Full immersion. In this research, down milling method was employed in end milling due to some advantages like better surface finish, less heat generation, larger tool life and better machining accuracy.

Coding of the independent variables: The independent variables at different levels were coded taking into considerations the limitation and capacity of the cutting tools. Levels of independent and coding identification are presented in Table 2, for experiment using Coated TiN inserts, respectively.

Experimental design: The design of the experiments (Design-Expert Software, 2000) has an effect on the number of experiments required. Therefore, it is important to have a well-designed experiment to minimize the number of experiments which often are carried out randomly. For this reason, a full CCD with 2 blocks and 5 replication of centre point in each factorial block was selected to design the experiments. The Central Composite Design (CCD) is the most popular Response Surface Method (RSM) design. Central composite designs require 5 levels of each factor: $-\alpha$, $-1,0,1$ and $+\alpha$ which are called coded values of the factors. This experimental design provides 5 levels for each of the independent variables. This ultimately resulted in 15 experiments, with 4 factorial points, 6 axial points and 5 centre points. Cutting conditions in coded factors and the chip serration frequency values for machining S45C material obtained using TiN coated cemented carbide insert are presented in Table 3.
Table 2: Coding Identification for end milling using Coated TiN insert

\begin{tabular}{lrrrrr}
\hline $\begin{array}{l}\text { Level of } \\
\text { coding }\end{array}$ & $\begin{array}{c}\text { Lowest } \\
-\sqrt{2}\end{array}$ & \multicolumn{1}{c}{\begin{tabular}{c} 
Low \\
\multicolumn{1}{c}{-1}
\end{tabular}} & $\begin{array}{c}\text { Centre } \\
0\end{array}$ & $\begin{array}{l}\text { High } \\
+1\end{array}$ & $\begin{array}{c}\text { Highest } \\
+\sqrt{2}\end{array}$ \\
\hline $\begin{array}{l}\mathrm{x}_{1} \text { cutting speed, } \\
\text { v m min }\end{array}$ & 100.000 & 114.25 & 158.000 & 218.50 & 250.000 \\
$\begin{array}{l}\mathrm{x}_{2} \text { axial depth } \\
\text { of cut, mm }\end{array}$ & 1.005 & 1.15 & 1.590 & 2.20 & 2.516 \\
$\begin{array}{l}\mathrm{x}_{3} \text { feed, } \\
\text { mm/tooth }\end{array}$ & 0.039 & 0.05 & 0.089 & 0.16 & 0.204 \\
\hline
\end{tabular}

Table 3: Chip serration frequency results and cutting conditions in

\begin{tabular}{|c|c|c|c|c|c|}
\hline \multirow{2}{*}{$\begin{array}{l}\text { Std. } \\
\text { No. }\end{array}$} & \multirow[b]{2}{*}{ Type } & \multicolumn{3}{|c|}{ Level of coding } & \multirow{2}{*}{$\begin{array}{l}\text { Chip serration } \\
\text { frequency }(\mathrm{Hz})\end{array}$} \\
\hline & & $\mathrm{x}_{1}$ & $\mathrm{x}_{2}$ & $\mathrm{x}_{3}$ & \\
\hline 1 & Fact & 1.00 & 1.00 & -1.00 & 4761.884 \\
\hline 2 & Fact & 1.00 & -1.00 & 1.00 & 17144.150 \\
\hline 3 & Fact & -1.00 & 1.00 & 1.00 & 2669.419 \\
\hline 4 & Fact & -1.00 & -1.00 & -1.00 & 7836.639 \\
\hline 5 & Centre & 0.00 & 0.00 & 0.00 & 8286.909 \\
\hline 6 & Centre & 0.00 & 0.00 & 0.00 & 8572.664 \\
\hline 7 & Centre & 0.00 & 0.00 & 0.00 & 8001.153 \\
\hline 8 & Centre & 0.00 & 0.00 & 0.00 & 8286.909 \\
\hline 9 & Centre & 0.00 & 0.00 & 0.00 & 8001.153 \\
\hline 10 & Axial & -1.41 & 0.00 & 0.00 & 3647.301 \\
\hline 11 & Axial & 1.41 & 0.00 & 0.00 & 10788.010 \\
\hline 12 & Axial & 0.00 & -1.41 & 0.00 & 8661.566 \\
\hline 13 & Axial & 0.00 & +1.41 & 0.00 & 5448.404 \\
\hline 14 & Axial & 0.00 & 0.00 & -1.41 & 5329.961 \\
\hline 15 & Axial & 0.00 & 0.00 & +1.41 & 4624.640 \\
\hline
\end{tabular}
coded factors

Cutting experiments were carried out in a block of S45C. The work-piece material was clamped onto the machine table to provide maximum rigidity. The transforming equations are given below:

$$
\begin{aligned}
& \mathrm{x}_{1}=\frac{\ln \mathrm{V}-\ln 158}{\ln 218.5-\ln 158} \\
& \mathrm{x}_{2}=\frac{\ln \mathrm{a}-\ln 1.59}{\ln 2.2-\ln 1.59} \\
& \mathrm{x}_{3}=\frac{\ln \mathrm{f}_{\mathrm{z}}-\ln 0.089}{\ln 0.16-\ln 0.089}
\end{aligned}
$$

\section{RESULTS AND DISCUSSION}

Development second order model using CCD design: Fit and summary test in Table 4 summarizes that the quadratic model CCD models was more significant than linear model and it also proved that linear model has a significant Lack Of Fit (LOF). Therefore, the quadratic model was chosen in order to develop the CCD model. The second order quadratic Chip Serration Frequency model is given as:

$$
\begin{aligned}
\hat{y}_{2}= & 8.94+0.36 x_{1}-0.16 x_{2}-0.05 x_{3}-0.058 x_{1}^{2} \\
& -0.013 x_{2}^{2}-0.18 x_{3}^{2}-0.10 x_{1} x_{2}+0.43 x_{1} x_{3}
\end{aligned}
$$


Am. J. Engg. \& Applied Sci., 3 (1): 193-200, 2010

Table 4: Fit and summary test of the second order CCD model

Sequential model sum of squares

\begin{tabular}{llllrrl} 
& $\begin{array}{l}\text { Sum of } \\
\text { Source }\end{array}$ & DF & $\begin{array}{l}\text { Mean } \\
\text { square }\end{array}$ & F-value & Prob>F & \\
\hline Mean & 1167.38 & 1 & 1167.38 & & & \\
Block & 0.16 & 1 & 0.16 & & & \\
Linear & 2.18 & 3 & 0.73 & 11.42 & 0.0014 & Suggested \\
2FI & 0.39 & 3 & 0.13 & 3.59 & 0.0741 & \\
Quadratic & 0.25 & 3 & 0.082 & 98.54 & 0.0003 & Suggested \\
Cubic & 0.000 & 0 & & & & Aliased \\
Residual & $3.349 \mathrm{E}-003$ & 4 & $8.382 \mathrm{E}-004$ & & & \\
Total & 1170.35 & 15 & 78.02 & & & \\
\hline
\end{tabular}

Table 5: Analysis Of Variance (ANOVA) of quadratic CCD model

\begin{tabular}{llclrrl}
\hline Source & $\begin{array}{l}\text { Sum of } \\
\text { squares }\end{array}$ & DF & $\begin{array}{l}\text { Mean } \\
\text { square }\end{array}$ & \multicolumn{1}{l}{$\begin{array}{l}\text { v } \\
\text { value }\end{array}$} & Prob>F & Remarks \\
\hline Block & 0.16 & 1 & 0.16 & & & \\
Model & 2.81 & 8 & 0.35 & 249.49 & $<0.0001$ & significant \\
$\mathrm{X}_{1}$ & 1.05 & 1 & 1.05 & 743.42 & $<0.0001$ & \\
$\mathrm{X}_{2}$ & 0.11 & 1 & 0.11 & 76.23 & 0.0003 & \\
$\mathrm{X}_{3}$ & 0.010 & 1 & 0.010 & 7.15 & 0.0442 & \\
$\mathrm{X}_{1} 2$ & 0.026 & 1 & 0.026 & 18.15 & 0.0080 & \\
$\mathrm{X}_{2} 2$ & $1.258 \mathrm{E}-003$ & 1 & $1.258 \mathrm{E}-003$ & 0.89 & 0.3881 & \\
$\mathrm{X}_{3} 2$ & 0.23 & 1 & 0.23 & 163.56 & $<0.0001$ & \\
$\mathrm{X}_{1} \mathrm{X}_{2}$ & 0.020 & 1 & 0.020 & 14.53 & 0.0125 & \\
$\mathrm{X}_{2} \mathrm{X}_{3}$ & 0.36 & 1 & 0.36 & 257.02 & $<0.0001$ & \\
Residual & $7.047 \mathrm{E}-003$ & 5 & $1.409 \mathrm{E}-003$ & & & \\
Lack of fit & $3.699 \mathrm{E}-003$ & 1 & $3.699 \mathrm{E}-003$ & 4.42 & 0.1034 & Not significant \\
Pure error & $3.349 \mathrm{E}-003$ & 4 & $8.372 \mathrm{E}-004$ & & & \\
Cor total & 2.98 & 14 & & & & \\
\hline
\end{tabular}

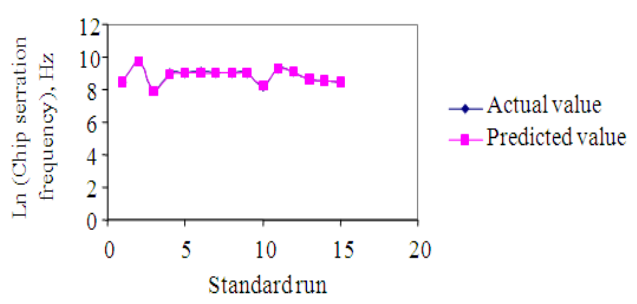

Fig. 5: Chip serration frequency contours of experimental and quadratic CCD predicted values

Figure 5 shows the contours of actual results and the predicted values of quadratic CCD models. The graphs indicated that the quadratic model leads to closer results to the actual values. To verify the adequacy of the proposed second order CCD model, ANOVA was used and the results are shown in the Table 5. The quadratic CCD model shows that cutting speed has the most significant effect on chip serration frequency, followed by axial depth of cut and feed.

The normal probability plots of the residuals and the plots of the residuals versus the predicted response for chip serration frequency are shown in Fig. 6 and 7 respectively. A check on the plots in Fig. 6 revealed that the residuals generally fall on a straight line implying that the errors are distributed normally. Also Fig. 7 revealed that they have no obvious pattern and unusual structure. This implies that the models proposed are adequate and there is no reason to suspect any violation of the independence or constant variance assumption.

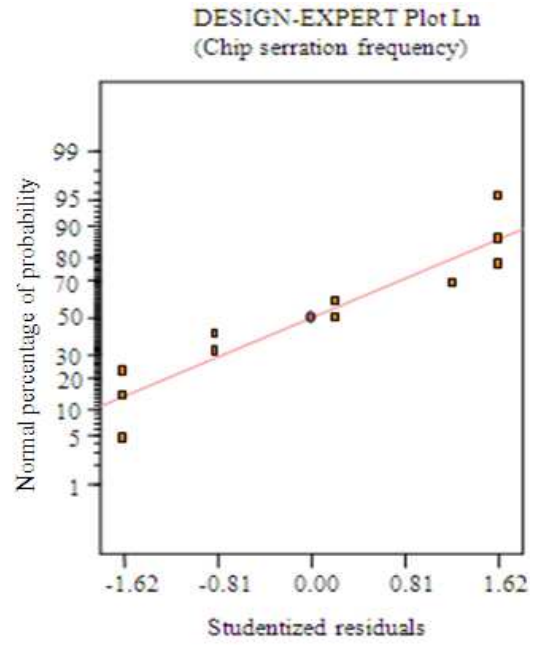

Fig. 6: Normal probability plot of residuals for fc data

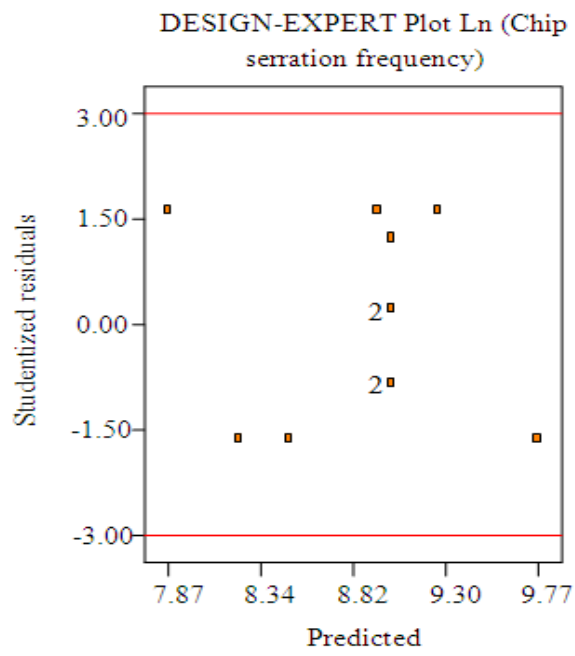

Fig. 7: Plot of residuals Vs predicted response for fc data

Analysis of model: For the analysis of the developed quadratic model, a Matlab code was developed to represent the individual cutting parameters effects on chip serration frequency. Figure $8 \mathrm{a}$ shows that with the increase of cutting speed the chip serration frequency increases and these trends follow for the other cases also but along with the increase of feed the chip serration also decreases.

Figure $8 \mathrm{~b}$ shows that with the increase of cutting speed the chip serration frequency increases and these trends follow for the other cases also but along with the increase of depth of cut the chip serration also decreases. The contours affirm that chip serration frequency is affected by the cutting speed followed by axial depth of cut and feed. 


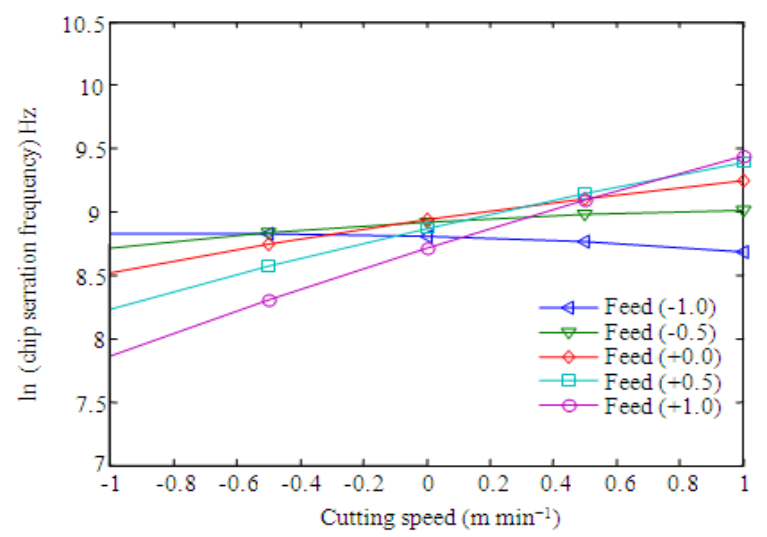

(a)

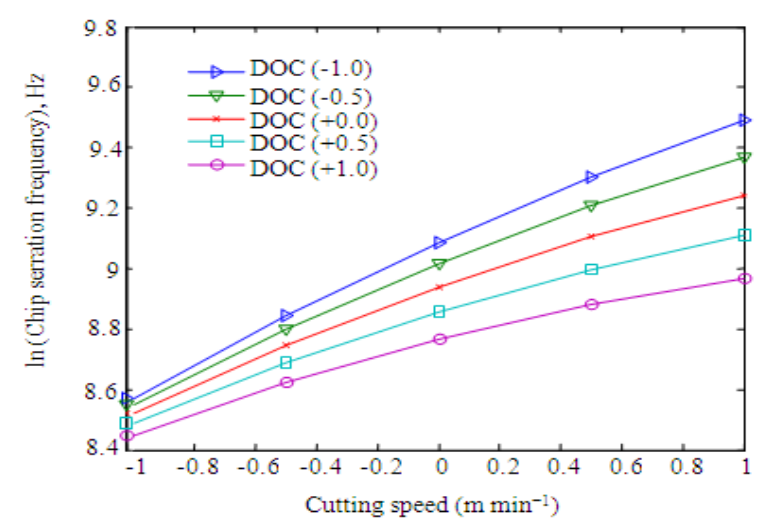

(b)

Fig. 8: Effect of cutting speed on chip serration frequency at (a): Effect of cutting speed on chip serration at different feed with DOC fixed (0.0 levels) (b): Effect of cutting speed on chip serration at different DOC at Fixed Feed ( 0.0 coded $)$

\section{CONCLUSION}

This study discussed the instabilities of the chip formation and development of a mathematical model for the prediction of the instability process. An analytical approach was developed for defining the chip formation instability in terms of chip serration frequency. Based on the experimental findings a statistical model for the prediction of chip serration frequency secondary in end milling of S45C using coated TiN insert was developed.

The two-stage effort for obtaining a chip serration model by surface response methodology and validation of this model by experiment has resulted in a fairly useful method of obtaining process parameters in order to predict the chip serration frequency.
The developed quadratic CCD model indicate that the cutting speed was the most significant influence on chip serration frequency, followed by depth of cut and feed

An increase in either the feed or the axial depth of cut decreases the chip serration frequency, whilst an increase in the cutting speed increases the chip serration frequency.

The CCD model developed by RSM using Design Expert package are able to provide accurately predicted values of chip serration close to actual values found in the experiments. The equations are checked for their adequacy with a confidence level of $95 \%$.

\section{REFERENCES}

Altintas, 1991. End milling force algorithms for CAD systems. Ann. CIRP., 40: 31-34. http://cat.inist.fr/?aModele $=$ afficheN\&cpsidt $=5403$ 479

Amin, A.K.M.N. and N.V. Talantov, 1986. Influence of the instability of chip formation and preheating of work on tool life in machining high temperature resistant steel and titanium alloys. Mech. Eng. Res. Bull., 9: 52-62.

Amin, A.K.M.N., 1982. Investigation of the laws governing the formation of chatter during metal cutting processes and the influence of chatter on tool wear. Ph.D. Thesis, Georgian Polytechnic Institute, Georgia, USSR, pp: 261.

Amin, A.K.M.N., 1983. Investigation of the mechanism of chatter formation during metal cutting process. Mech. Eng. Res. Bull., 6: 11-18.

Astakhov, V.P. and S.V. Shvets, 2001. A novel approach to operating force evaluation in high strain rate metal-deserration technological processes. J. Mater. Process. Technol., 117: 226-237. DOI: $10.1016 / \mathrm{S} 0924-0136(01) 01103-7$

Design-Expert Software, 2000. Version 6.0.8, User's Guide, Technical Manual. Stat-Ease Inc., Minneapolis, MN.

Ekinovic, S., S. Dolinsek, S. Brdarevic and J. Kopac, 2002. Chip formation process and some particularities of high-speed milling of steel materials. Trends in the Development of Machinery and Associated Technology, TMT, B\&H, Neum.

Elbestawi, M.A., 1997. High speed milling of dies and molds in their hardened state. Ann. CIRP., 46: 57-62. http://direct.bl.uk/bld/PlaceOrder.do?UIN=028292 $340 \&$ ETOC $=$ RN\& from $=$ searchengine 
Komanduri, R. and B.F. von Turkovich, 1981. New observations on the mechanism of chip formation when machining titanium alloys. Wear, 69: 179181.

Komanduri, R., 1981. On the mechanism of chip serration in machining. J. Eng. Ind. 103: 33-51.

Komanduri, R., T. Schroeder, 1982. On the catastrophic shear instability of high speed machining of an AISI 4340 steel. J. Eng. Ind., 104: 121-131.

Lindberg, B. and B. Lindstrom, 1983. Measurements of the segmentation frequency in the chip formation process. Ann. CIRP., 32: 17-20.

Minis, I., 1993. A new theoretical approach for the prediction of machine tool chatter in milling. ASME. J. Eng. Ind. 115: 1-1. http://direct.bl.uk/bld/PlaceOrder.do?UIN=004016 $671 \&$ ETOC $=$ EN\& from=searchengine

Montgomery, D.C., 1997. Design and Analysis of Experiments. 6th Edition., Wiley, New York, ISBN: 0444820612, pp: 1229.

Ning, Y., M. Rahman and Y.S. Wong, 2001. Investigation of chip formation in high speed end milling. J. Mater. Process. Technol., 113: 360-367. DOI: 10.1016/S0924-0136(01)00628-8

Smith, S. and J. Tlusty, 1987. Update on high-speed milling dynamics. ASME Prod. Eng. Div., 25: 153-165.

Talantov, N.V., 1984. Plastic Deformation and SelfExited Vibrations in Metal Cutting (in Russian). In: Metalworking Technology and Automatization of Manufacturing Processes, Talantov, N.V. (Ed.). VPI, Volgograd, pp: 37-41.
Talantov, N.V., A. Amin and N.P. Chereomushnikov, 1980. Temperature deformation laws of chatter formation during metal cutting process. Proceeding of the 5th Soviet National Conference Teplophysika Technologichieskikh Processov, Volgograd USSR., pp: 92.

Tlusty, J. and F. Ismail, 1983. Special aspects of chatter in milling. ASME. J. Acoust. Stress, Reliability, 105.

Toenshoff, H.K., 1969. Chip formation at high cutting speed. ASME Winter Meeting.

Tonshoff, H.K., P.B. Amor and P. Amdrae, 1999. Chip formation in High Speed Cutting (HSC). SME Paper MR99-253.

Trent, E.M., 2000. Metal Cutting. Oxford Limited, London, ISBN: 075067069X, pp: 446. 